\title{
Modeling the Influence of Action on Spatial Attention in Visual Interactive Environments
}

\author{
Ali Borji, Dicky N. Sihite, and Laurent Itti
}

\begin{abstract}
A large number of studies have been reported on top-down influences of visual attention. However, less progress have been made in understanding and modeling its mechanisms in real-world tasks. In this paper, we propose an approach for learning spatial attention taking into account influences of physical actions on top-down attention. For this purpose, we focus on interactive visual environments (video games) which are modest real-world simulations, where a player has to attend to certain aspects of visual stimuli and perform actions to achieve a goal. The basic idea is to learn a mapping from current mental state of the game player, represented by past actions and observations, to its gaze fixation. A data-driven approach is followed where we train a model from the data of some players and test it over a new subject. In particular, two contributions this paper makes are: 1) employing multimodal information including mean eye position, gist of a scene, physical actions, bottom-up saliency, and tagged events for state representation and 2) analysis of different methods of combining bottom-up and top-down influences. Comparing with other top-down task-driven and bottom-up spatio-temporal models, our approach shows higher NSS scores in predicting eye positions.
\end{abstract}

\section{INTRODUCTION}

The concept of saliency has attracted a lot of attention over the past several years. Basically, it is a fast and lowcost pre-processing step to select important image regions or objects to pass to higher-level and computationally demanding processes.

The main concern in modeling saliency is how, when, and based on what, to select salient image regions. It is often assumed that attention is attracted by salient stimuli or events in the visual array [1][2]. While this is the case, it is also known that a larger portion of attentional behavior comes from ongoing task inferences which dynamically change and are dependent on the algorithm of the task. Computational modeling of task influences on attention is conceptually hard to frame. The biggest challenge comes from the fact that we don't know much about how humans perform complex tasks. This has been at the focus of artificial intelligence (AI) and cognitive science research for the past 50 years. However, we know to some extent about algorithms and attentional behaviors of some laboratory-scale stimuli and tasks. One solution when dealing with complex problems is learning from data, experiences or history which could be gathered from the behavior of other humans especially when the goal is to explain human data.

There are already many bottom-up saliency models for static (still images) and spatio-temporal stimuli (videos).

Authors are with the Department of Computer Science, University of Southern California, Los Angeles, USA. \{borji, sihite, itti\}@usc.edu
However, bottom-up models are inflexible (Fig. I) and can account for only a small fraction of the observed fixations in natural behavior [4][5][6]. Our goal in this study is to introduce a top-down spatial attention model which could automatically direct gaze based on task. Instead of trying to figure out an explicit algorithm for doing a task, (e.g., designing a state space and mapping its states to actions and attended locations), we are following a data-driven approach which could be easily applied to any task and situation.

\section{A. Bottom-up (BU) models}

The bottom-up saliency assumption is based on the hypothesis that certain features of the visual scene inherently attract gaze. That is, that vision is essentially reactive and stimulus driven. Typically, multiple low-level visual features such as intensity, color, orientation, texture, and motion are extracted from an image at multiple scales. Saliency maps are computed for different features which are then normalized and combined in a linear or non-linear fashion into a master map that represents the conspicuity of each pixel [16].

Our work in this paper falls in the category of saliency models based on machine learning approaches. Some models train a classifier to distinguish fixated patches from random patches. Facing a scene they assign a value to each patch that is the probability of that patch to be fixated. Kienzle et al. [7] learned a model of saliency directly from human eye movement data. Their model consists of a nonlinear mapping from a normalized image patch to a real value, trained to yield positive values on fixated patches, and negative values on randomly selected image patches. Judd et al. [8] used a SVM classifier for an attention model based on low-, mid and high-level features calculated by existing saliency methods. In modeling eye fixations of observers when looking for a pedestrian in a scene, Ehinger et al. [26] showed that a model of search guidance combining three sources: low-level saliency, target features, and scene context, outperforms models based on any of these single sources. Vig et al. [9] used 3D spatio-temporal volumes from video for spatiotemporal saliency modeling. Li et al. [10] proposed a multi-tasking Bayesian approach for combining bottomup and top-down saliency components. Kimura et al. [11] learned a Dynamic Bayesian Network (DBN) to predict the likelihood of locations where humans typically focus on a video scene. Chikkerur et al. [27] presented a Bayesian model based on assumptions that the goal of the visual system is to say what is where and visual processing happens sequentially. 

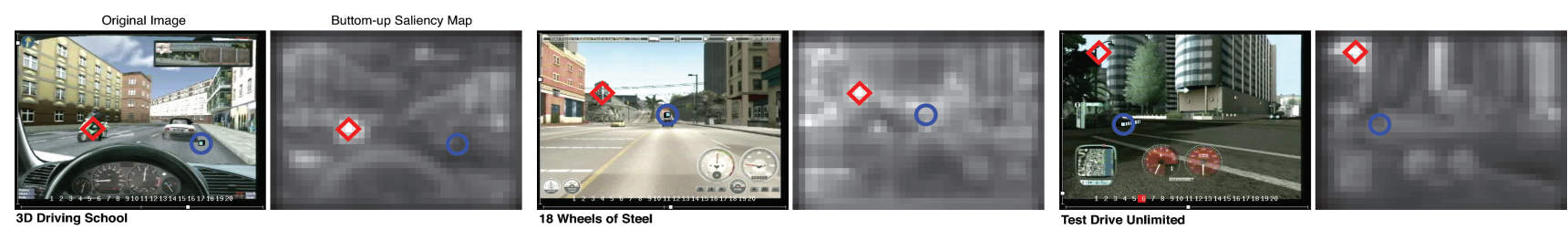

Fig. 1. Sample frames from our game stimuli and their corresponding BU saliency maps [16]. Red diamond shows the maximum of the saliency map and blue circle is the actual eye position. Left and bottom bars in frames are the pedal and wheel positions, respectively. Actions are represented by numbers at the bottom. Attention is attracted to task-relevant regions which do not agree with the BU saliency.

\section{B. Top-down (TD) models}

The other main component of attention comes from topdown demands such as knowledge of the task, emotions, expectations, predictions, etc. which are embedded in a temporally extended task. Modeling top-down attention is hard because: 1) it is difficult to frame and conceptually define the problem, 2) different tasks require different algorithms, and 3 ) there is a high degree of inter-subject variability. In this paper, we take another step (after [15]) in modeling topdown spatial attention considering multi-modal information including physical actions.

Research on top-down attention dates back to the classic study of Yarbus [3] who showed that gaze patterns are dependent on the asked question when viewing a picture. Research on task-driven influences of gaze have been mostly at the analysis level. It has been shown that the vast majority of fixations are directed to task-relevant locations, and fixations are coupled in a tight temporal relationship with other task-related behaviors such as reaching and grasping [12]. Furthermore, eye movements often provide a clear window to the mind of an observer in a way that it is sometimes possible to infer how a subject solves a particular task from the pattern of his/her eye movements for tasks like "block copying" [13] , "making tea" [4] , "driving" [14], etc.

In [15], Peters and Itti learned a mapping using gist of a scene to eye fixation from data of subjects playing a video game. In [17], using this model, they showed that during the occurrence of an event (such as hitting a target in shooting games or accident in driving games) bottom-up cues are more important than top-down cues and based on this proposed event detectors by combining stimuli and behavior signatures. While this model is interesting, it does not benefit from the real potential of interactive environments which are interactions via physical actions. In our work, we follow a similar fashion by proposing a richer state representation and propose a new approach to combine bottom-up and topdown cues. In a related study, Navalpakkam and Itti [18] tried to build a top-down attention framework in conjunction with the saliency model in situations where the algorithm for the task is at hand. Sprague and Ballard [19], proposed a method based on reinforcement learning for learning visiomotor behaviors and used their model to account for saccades in a side walking task.

\section{Influence of action on attention}

The interaction between action and perception makes up one of the most important facets of our daily life. Many stud- ies support the idea that perception affects action (e.g., [20]). It has also been proposed that changes due to actions lead to corresponding changes in perception [20][21]. An example of interaction between action and perception is driving which also needs sophisticated attentional behavior. In [23], authors showed that preparation of a grasping movement affects detection and discrimination of visual stimuli. Our work also borrows from the ideas of sensory-motor integration: The process by which the sensory and motor systems communicate and coordinate with each other (e.g., hand-eye coordination). The above statement is closely related to the premotor theory of spatial attention which argues that the major function of attentional selection is not only reducing the incoming information, but rather to select a proper action on the basis of a specific stimulus [22].

\section{Our approach and contributions}

We aim to learn top-down spatial attention (where to look) from visual information and physical actions recorded from human subjects playing video games. The basic idea is to best estimate the mental state of the player and map it to an eye fixation. For state estimation, we merged all information including scene gist, physical actions, salient regions, and events. A classifier is learned from these data and is used to predict the eye fixations of a new subject.

A central open question in saliency modeling is "how the bottom-up salient and top-down task-driven stimuli are integrated in the course of a task"? We tackled this question by evaluating different ways of integrating $\mathrm{BU}$ and TD attention components either in the decision space or at sensory level. Experiments were performed on driving which is a daunting task demanding high-level sensory-motor integration and attention/action coordination skills. It has also been the subject to several behavioral and computational modeling studies (e.g., [14][25]).

Our model: 1) is easily applicable to interactive visual environments when subjects perform physical (motor) actions and visually attend and 2) has potential applications in robotics and computer vision including robot localization, interactive computer graphics environments ("virtual reality" or video games), flight and driving simulators (and assistants), as well as visual prosthetic devices.

\section{PSYCHOPHYSICS AND EYE TRACKING}

To set a basis and benchmark for future research and large-scale quantitative evaluation of studies on task-driven top-down saliency modeling, we have collected a large scale dataset of videos along with eye tracking data and actions. 

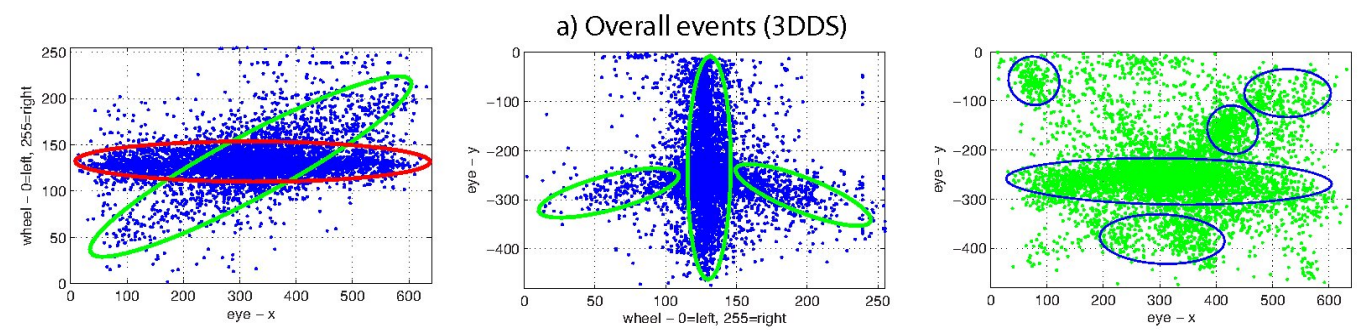

b) Event-based (3DDS)

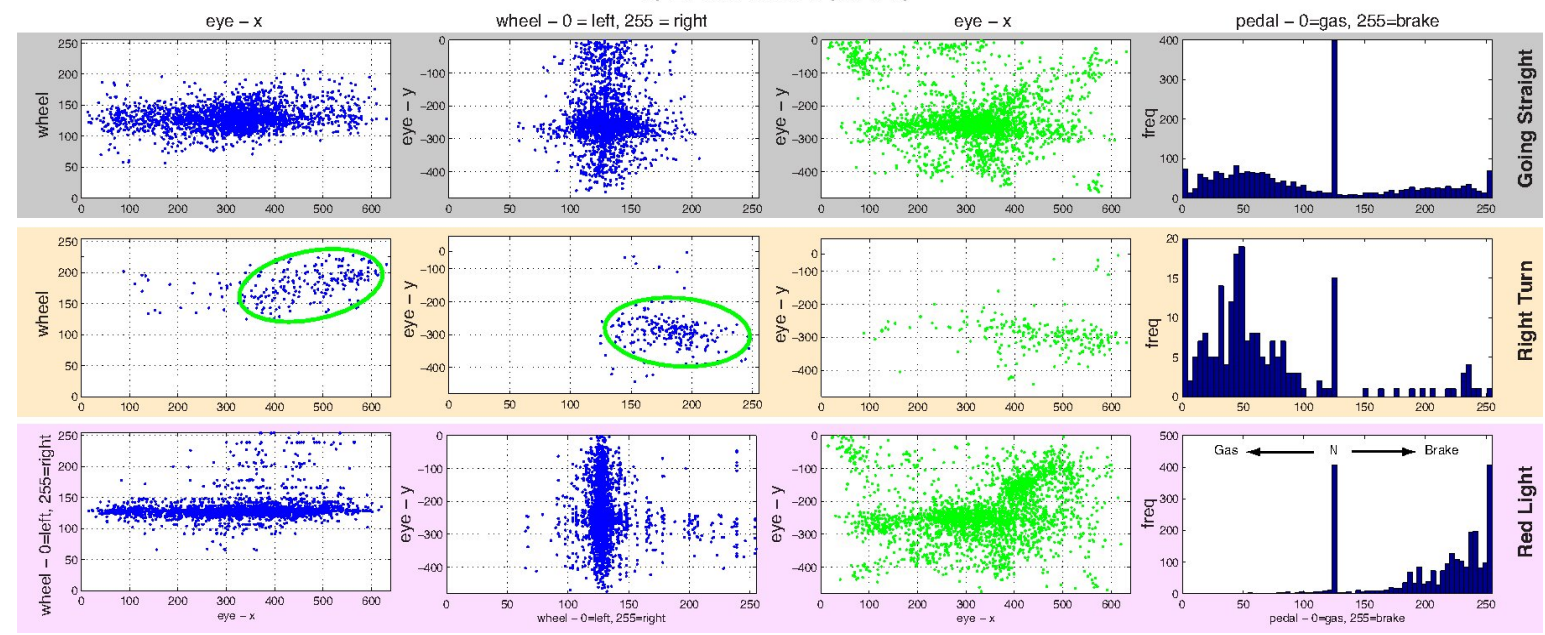

Fig. 2. a) Correlation between wheel and eye-x and eye-y saccade coordinates (left two panels), and saccade positions (right panel) overall all events of 3DDS. b) Left 3 panels: same as (a) for sample events. Right panel shows the frequency of pedal positions.

Accompanying code in $\mathrm{C}++$ and Matlab will be available on the web to facilitate future research.

\section{A. Data collection}

Participants were 10 subjects between 18-25 years old with valid driving license and at least 2 years of driving experience. They had normal or corrected-to-normal vision and were compensated for their participation. Experimental protocol was approved by the university of Southern California Institutional Review Board. Each subject played each of the 3 games: 3D Driving School (3DDS), 18 Wheels of Steel (18 WoS), and Test Drive Unlimited (TDU) (Fig. I). There was a 5-min training session for each game in which subjects were introduced to the goal of the game, rules, buttons, etc. After training, subjects played the game for another 5 minutes. At the beginning of the test session, eye tracker was calibrated using 9-point calibration. Training and testing phases were from the same game but different situations. Subject's distance from screen was $130 \mathrm{~cm}$ yielding a field of view of $43^{\circ} \times 25^{\circ}$. The overall recording resulted in 2.5 hours of $156 \mathrm{~GB}$ video, 192,000 frames, 1,536,000 fixations, and 10,518 saccades.

Subjects played driving games on PC1 which had Windows XP running the games. An array of wheel, pedal and other actions (signal, mirror, etc) was logged with frequency of $62 \mathrm{~Hz}$. The frames were recorded on $\mathrm{PC} 2$ running Linux Mandriva OS. Game stimuli were shown to the subject at $30 \mathrm{~Hz}$. This machine sent a copy of each frame to LCD monitor and saved one copy to the hard disk. PC2 also instructed the eye tracker (PC3) for recording eye positions when watching the screen. PC2 had a dualCPU processor and used SCHED_FIFO scheduling to ensure microsecond accurate timing. Each subject's right eye position was recorded at $240 \mathrm{~Hz}$ with a hardware-based eyetracking system (ISCAN Inc. RK-464). Subjects drove using the Logitech Driving Force GT steering wheel, automatic transmission, brake and gas pedals, 11-inch rubber-overmold rim, 900 degrees rotation (only 360 degrees; 180 left, 180 right; were used in experiments), Force Feedback, connected via USB to the PC1.

\section{B. Model-free analysis of the dataset}

We are interested in two types of model-free analyses: 1) analysis of saccade and fixation distributions in order to find out which locations attract subject's attention; and 2) correlation among eye fixation and actions to be used for eye movement prediction later.

Fig. 2.a shows task-relevant locations for all 10 subjects (each dot represents one saccade) over 3DDS. Since fixation maps are highly dense, only saccades are shown for illustration purpose. However, reported patterns are the same over both fixations and saccades. Over 3DDS, task-relevant regions are: an arrow sign at the top-left indicating direction, instruction command at top, instructor and rear-view mirror at the top-right, horizontal view and road (middle), red light slightly above road, and interior (speedometer) of the car at the bottom shown by blue ellipses. Please see Fig. I the leftmost panel for a sample frame and Fig. 2.a the rightmost panel for saccade locations. As it shows, there is a strong horizontal bias in this task similar to free-viewing and visual 
search tasks [8]. Profile of wheel angle vs. eye-y (image width) shows that subjects viewed all the vertical line when wheel was released (value of 127). This happened when subjects were driving straight or were stopped. There is a slight tendency to look at the bottom when turning left or right. Wheel angle vs. eye-x(image height) shows two main saccade directions: 1) horizontal bias, and 2) diagonal which means that wheel toward right is correlated with saccade to the right (similarly wheel toward left correlates with saccade to the left).

To further analysis the data we also tagged each frame of games based on different events that happened in driving. Some games did not have all the events. Events of each game could be found in Fig. 4. In "going straight" event, there is a horizontal and vertical bias (wheel vs. eye) and subjects looked more at the center (center-bias) while looking around to get important information (eye-x vs. eye-y). For "turn right" event, there is a rightward shift of fixations based on wheel (similarly for turn left). For "red light" event, since task-driven influences are not much strong (stopping situation), then subjects have time to look around (i.e., less task demand). Frequency of brake/gas is shown in right panels of Fig. 2.b. There is a peak at the center indicating that most of the time, gas/brake pedal is released. Subjects pressed the gas and brake more when turning right and at the red light, respectively.

To learn about the temporal relationships between eye position and wheel angle, we plotted fixations in steps of 32 of wheel angle for all games in Fig. 3. Green circle shows the mean eye fixation position and vertical red line shows the normalized (linearly to its max) wheel position. It could be seen that a linear relationship with the eye fixation holds for wheel positions between 64 to 192, but for extreme values it seems that wheel position is slightly leading. Temporal analysis along with tagged events, could help better fixation prediction provided that the event could be predicted correctly.

Fig. 4, shows the average number of saccades per frame of each event of all three games. It shows more saccades happen in "red light" and "mistakes" events and less in "turning" and "going straight". This along with sparseness of saccades/fixations for an event indirectly is a measure of how demanding is a behavior (event) and could be used as a cue for weighting top-down and bottom-up saliency maps.

\section{LEARNING TASK-DEPENDENT SPATIAL MAP}

In the following, we explain our model for learning taskdriven influences on eye position. First, we compiled a training set containing feature vectors and eye positions corresponding to individual frames from several video game clips. The learned mapping is then used to predict fixation location over a new frame. Note that here we are only interested in prediction of fixations (one fixation per frame). We compared two combination approaches: In the first approach called "decision combination method", individual predictors were learned by mapping a feature vector describing state or scene to corresponding fixation of that frame. Outputs of these

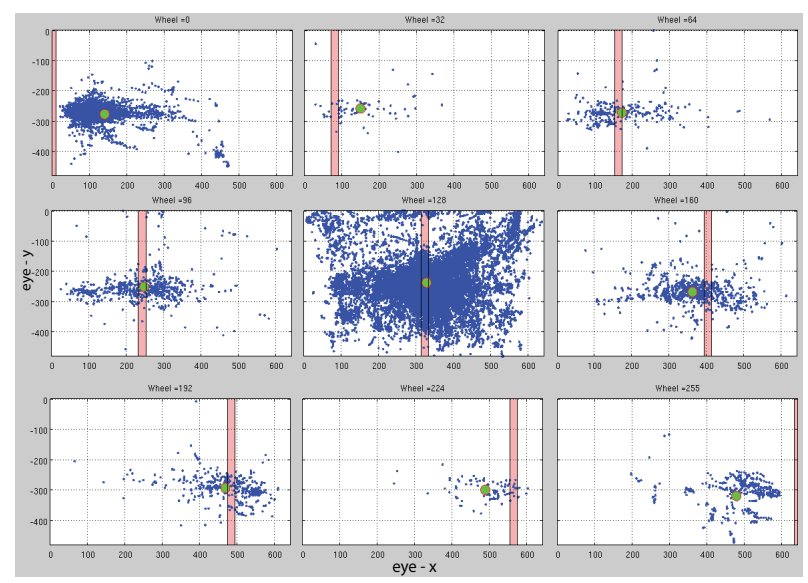

Fig. 3. Mean fixation position vs. linearly estimated steering wheel position over all games and events.

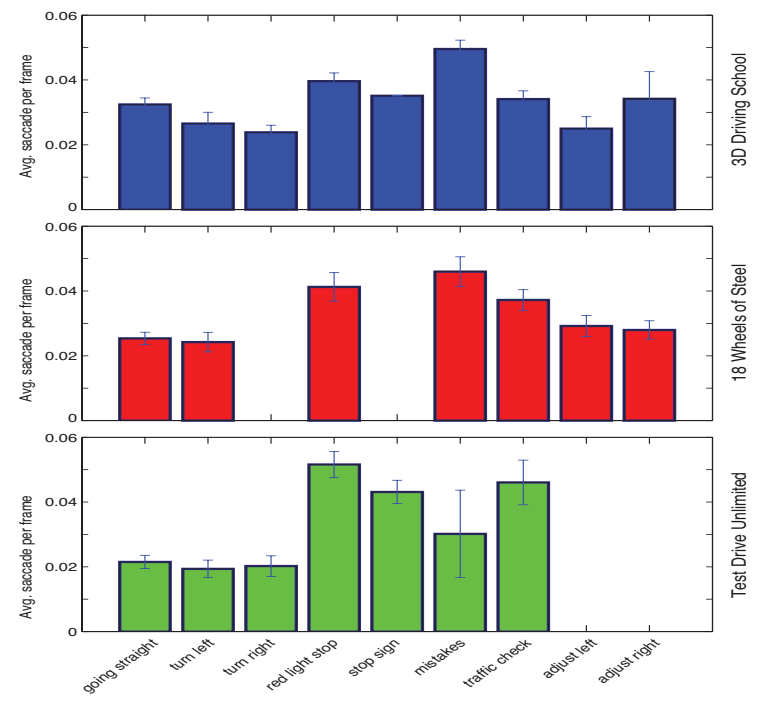

Fig. 4. Average number of saccades per frame for each event. Error bars are standard deviations.

predictors were then fused using addition or multiplication. In the second approach, "feature combination method", all feature vectors were combined in a single vector in a hope that it might give a better scene/state description. Then a mapping was learned from this vector to eye fixations.

\section{A. Training}

Let $S_{t}$ be the state of a player at time $t$ defined as $S_{t}=\left[b, L_{t-m}, \ldots L_{t-1}, L_{t}\right]$ as a history of past scene representations where $L_{t}=\left[G_{t}, B_{t}, A_{t}, E_{t}\right]$ is the information at time $t . b$ is a scalar bias value, $G_{t}$ is the gist of the scene, $B_{t}$ is the raw bottom-up saliency map, $A_{t}$ is the associated action for frame $t$ and $E_{t}$ is the labeled event. $m$ is the depth of history. When a task is Markovian, all information regarding state is available at the current time (i.e., $m=0$ ). We are interested in finding a mapping $\mathcal{F}$ from $S_{t}$ to $P_{t+1}=\left[x_{t+1}, y_{t+1}\right]$, the eye position at time $t+1$. Assume $q$ subjects have performed task $T$. Our collected data is $D=\{M, N\}$ where $M$ is a $n \times|S|$ matrix of feature vectors and $N$ is the $n \times|P|$ matrix of eye positions. $n$ is the number of data point from $q-1$ subjects and $|P|$ is 2 .. 
A classifier is learned from these data and is tested over the remaining $q$-th subject using leave-one-out cross validation.

In the decision combination approach, we combine decisions of predictions learned from different features. That is, saliency map at time $t$, is a function $\mathcal{G}$ of different $\mathcal{F}$ mappings, (each $\mathcal{F}$ could be considered as a single behavior). This arbitration mechanism, $\mathcal{G}$, itself is task dependent and tells how different top-down factors should be integrated. Here, we tried two simple integration functions: addition and multiplication. Since final behavior is a combination of pure bottom-up and top-down influences, we considered pure bottom-up map $B_{t}$ as an individual predictor as well.

In the feature combination approach, we started with a simple classifier $\mathcal{F}_{0}$ by only considering the bias term $b$ and gradually added more features to it to build more predictive classifiers $\left\{\mathcal{F}_{1}, \mathcal{F}_{2}, \ldots\right\}$.

Assuming a linear relationship between feature vectors and eye fixations [15], we solve the equation $M W=N$. Solution to this equation is: $W=M^{+} N$, where $M^{+}$is the pseudo inverse of matrix $M$. When feature vector is $b$, the solution (predicted map) is simply the average of all eye position vectors in $N$. This classifier is called mean eye position (MEP). This way, we are solving a linear regression classifier with the least squares method. We used SVD to find the pseudo inverse of matrix $M$. An important point here is that we set singular values smaller than half of the biggest singular value to a small value (0.001) to avoid numerical instability.

Vector $P$ which is eye position over the $640 \times 480$ image is down sampled to $20 \times 15$ and transformed into a $1 \times 300$ vector with a 1 at the actual eye position and zeros elsewhere. In testing phase, in order to predict the eye position for a new frame of a subject first, a feature vector (as above) is extracted and then a saliency map is generated by applying the learned mapping. Maximum of this map could be used to direct attention. In combination (addition and multiplication), saliency maps are linearly normalized and combined to form a new saliency map.

\section{B. Features}

The features that we used for representation of scene $\left(L_{t}\right)$ are explained below.

Mean eye position (MEP) . MEP is the prediction when distribution of fixations is available (average of all fixations over the train data). In dynamic environments used in this paper, since frames are generated dynamically and there are few fixations per frame, aligning frames (in contrast to movies) is not possible. If a method could dynamically predict eye movements in a frame by frame basis, then achieving a higher accuracy than MEP is possible.

Gist of the scene $(G)$. Gist is a rough representation of a scene that does not contain much details about individual objects or semantics but can provide sufficient information for coarse scene discrimination (e.g., indoor vs. outdoor). The pyramid-based feature vector (pfx) [24], relies on 34 feature pyramids from the bottom-up saliency model: 6 intensity channels, 12 color channels (first 6 red/green and next 6 blue/yellow color opponency), and 16 orientations. For each feature map there are 21 values that encompass average values of various spatial pyramids: value 0 is the average value of the entire feature map, values 1 to 4 are the average values of each $2 \times 2$ quadrant of the feature map and values 5 to 20 are the average value for each of the $4 \times 4$ grids of the feature map leading to overall of $34 \times 21=714$ elements.

Bottom-up saliency map $(B)$. This model includes 12 feature channels sensitive to color contrast (red/green and blue/yellow), temporal luminance flicker, luminance contrast, four orientations $(0,45,90,135)$, and four oriented motion energies (up, down, left, right). After a center-surround difference operation and across scale competitions, a unique saliency map is created and subsampled to a $20 \times 15$ feature vector which is then linearized to a vector of $1 \times 300$ [16].

Physical actions $(A)$. In the driving experiment, actions are a 22D feature vector containing wheel positions, pedals (brake and gas), left and right signals, mirrors, left and right views, gear change, etc which are wheel buttons subjects used while playing.

Labeled events $(E)$. Each frame of games was manually labeled belonging to one of different events such as left turn, right turn, going straight, adjusting left, adjusting right, stop sign, etc (Fig. 4). Hence this is only a scalar feature.

\section{Model-BASEd RESUlts}

To quantify how well model predictions matched with observers' actual eye positions, we used the normalized scanpath saliency (NSS) metric, which is defined as the response value at the human eye position, $\left(x_{h}, y_{h}\right)$, in a model's predicted gaze density map that has been normalized to have zero mean and unit standard deviation.

In the first experiment, we trained our model over each separate game. Each game segment (per subject) has 8,000 frames. Training was done over $9 \times 8,000$ frames and the learned model was tested over the remaining subject. Final results are averaged over 10 subjects. Fig. 5.a shows NSS scores of models with single features and best answers for both combination approaches (addition and multiplication) for each individual game. Over three games, decision combination approach resulted in higher NSS scores. Saliency maps learned from gist features and the raw BU map were the most informative ones. Feature combination resulted in lower performance but still higher than all other single predictors. In agreement with previous results [15], the BU raw map resulted in the least performance (below 0.5) again indicating that BU saliency does not account for task-driven fixations. High NSS score for gist means that scene representation is a good predictor of subject's state. Compared with the model of [15] that multiplies raw bottom-up and the TD model learned from the Gist, our combination approach resulted in higher NSS scores. Multiplication approach proposed in [15] resulted in NSS smaller than 1. Using only action features, our model outperforms MEP and Gaussian models significantly indicating influence of action on prediction of top-down attention. Prediction based on only event feature 
a) Game by game
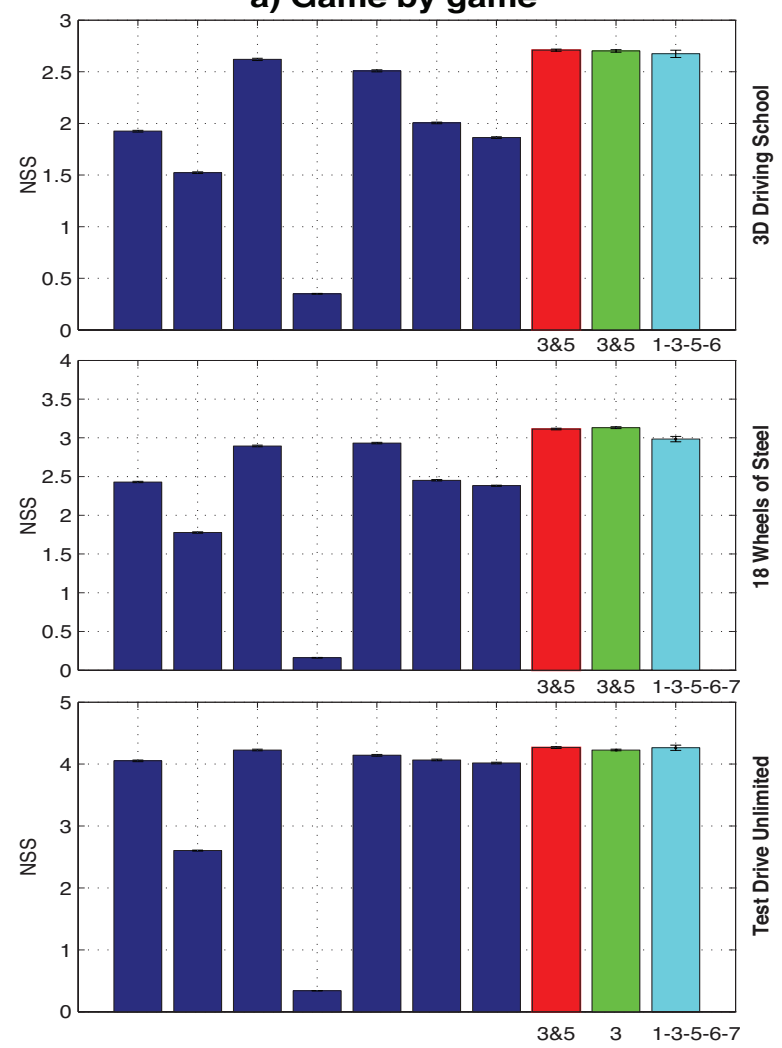

b) Over all games

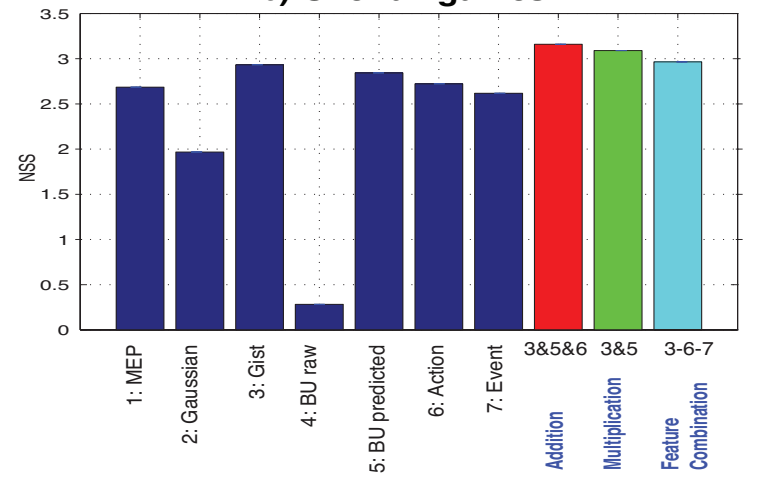

Fig. 5. NSS scores of different models with actual recorded eye positions (single features and combinations). a) model trained over each individual game, b) model trained over all games. A larger NSS score means a better fit. Each bar represents mean s.e.m. across all 80,000 fixations for each game. "\&" sign means that final maps were combined (decision combination) and "_. sign indicated that features were combined (feature combination).

was slightly lower than MEP but still better than Gaussian and raw BU map. All models performed significantly above chance $(N S S=0)$.

In the second experiment, we trained the model over all games, each time over 29 subjects and tested over the remaining subject. Results are shown in Fig. 5.b. Consistent with results in Fig. 5.a, decision combination approaches led to higher NSS performance. NSS values for MEP, Gaussian, action, event and gist in order are: 2.68, 1.96, 2.72, 2.61, and 2.93. Our results for addition, multiplication and Feature Combination are 3.16,3.08, and 2.96, respectively which are significantly higher than Gist model (paired t-test, $p<0.05$ ) across all fixations. It shows that our approach has more prediction power compared with previous models [15][16]. Action features alone are significantly higher than MEP and Gaussian. In feature combination approach, adding action and event features to the state representation improved the performance.

Fig. 6 shows sample frames from three games along with their corresponding predicted saliency maps from various models. Bottom-up saliency map (BU raw) shows spread activity with a weak maximum at the actual eye position. Predicted saliency maps by our models show dense activity at task relevant locations thereby narrowing attention and leading to higher NSS score. It seems that combined maps in general are more capable of finding the task-relevant regions. These maps change per frame as opposed to the static MEP and Gaussian.

High NSS score for Gaussian indicates high center-bias in these tasks which can be verified from mean eye positions (MEPs) in Fig. 7. The fact that the data is center-biased makes surpassing the MEP difficult (see Fig. 7). The main reason for this is that a huge number of fixations happen in the center which means MEP automatically has a lot of true positives. Given the high center bias, a predictor has only a small chance to show its superiority over few samples off the center.

\section{Discussion AND CONCLUSION}

In this work we analyzed the influence of action on visual attention and proposed general methods for modeling top-down spatial attention. Our models are more effective for tasks when attention is more influenced by motor actions. Results show that combining decisions works better than combining features for fixation prediction over driving games.

In our experiments, we noticed that the history depth $(m)$ is not helping much while adding computational complexity. Thus, we set $m$ to zero. This does not necessarily mean that history is not important. A more appropriate approach of employing history of previous actions and perceptions would be using sequence processing techniques such as hidden Markov models (HMM) and DBNs. The performance of our models is limited by the correlation among subjects in looking at the same spots for a same scene. The higher the correlation, the better learning and prediction.

A big issue in saliency modeling (either BU or TD) is handling center-bias. Most of the available datasets are center-biased meaning that a large proportion of fixations happen to be in the center of the image. For example, available still images shows photographer bias when photographers intentionally put interesting eye catching objects in the center [8]. Similarly, game designers dynamically change the viewpoint in order to put the needed object (main character, road, etc) at the center. This contaminates the scores. Gathering less center-biased datasets over movies and interactive setups and designing appropriate scores is very helpful for fair evaluation of top-down models in future.

One area to look for inspiration for designing more effective top-down models is recent video processing and analysis 


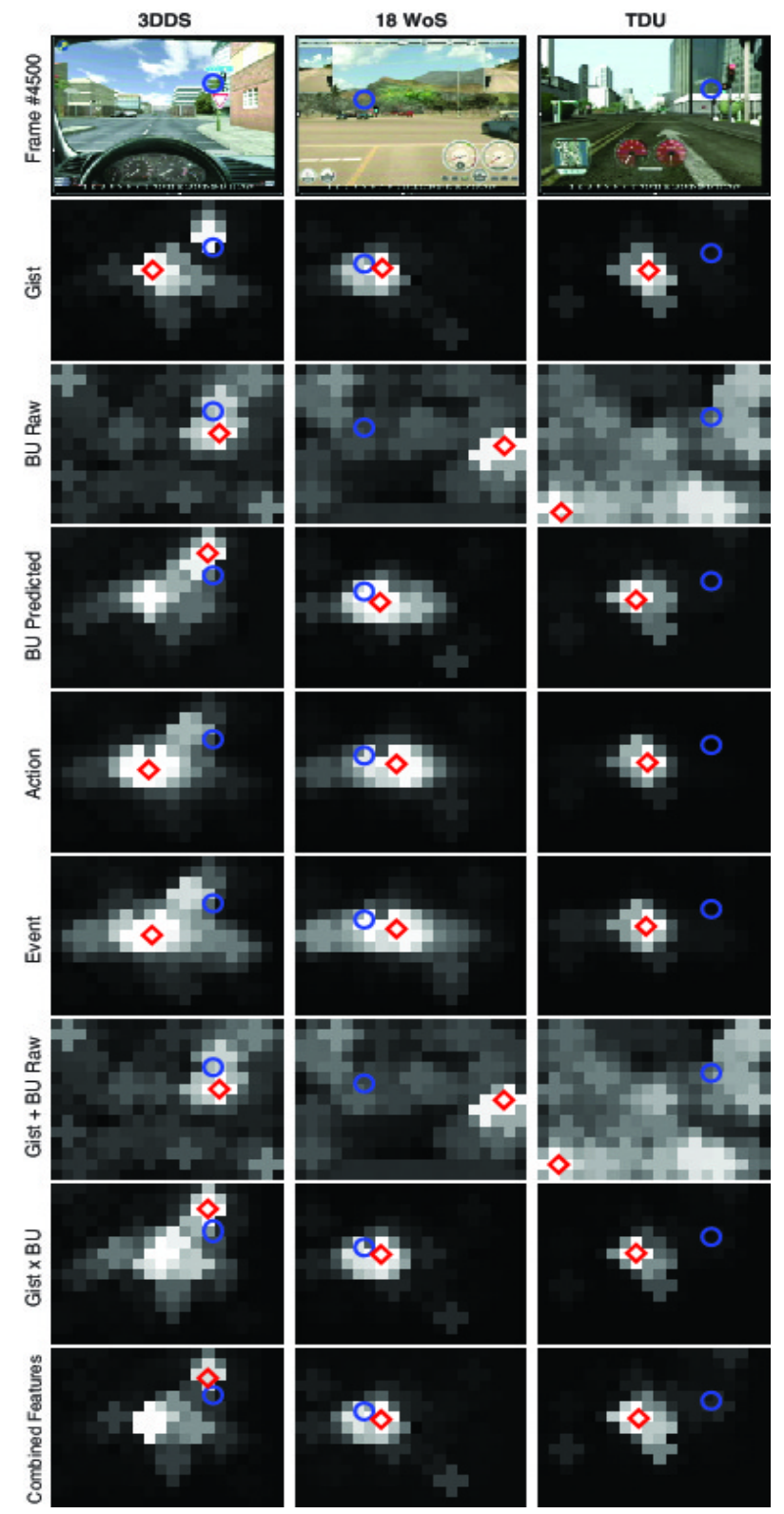

Fig. 6. Sample frames along with predicted top-down maps of different models. Each row is the output of a different model. BU raw is the output of the purely bottom-up saliency model and BU predicted means predicted saliency map when raw BU features are used. Red diamond: maximum of each map, blue circle: actual eye position for that frame. Gist $\times \mathrm{BU}$ is the point-wise product of $\mathrm{BU}$ predicted and gist models.
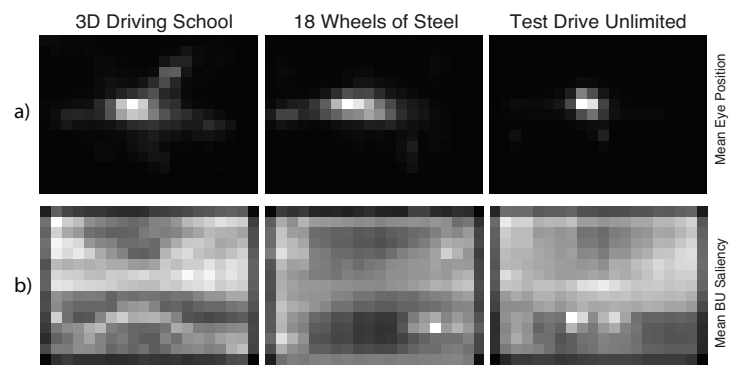

Fig. 7. a) Mean eye position maps, b) mean bottom-up saliency maps for three games. BU saliency maps have stable white regions which creates false positives over many frames. approaches (e.g., video segmentation, action recognition, and visual tracking). Here we followed a data-driven approach; a promising extension will be trying to infer some high-level knowledge or behaviors from data similar to [19].

Acknowledgments. This work was supported by Defense Advanced Research Projects Agency (DARPA).

\section{REFERENCES}

[1] L. Itti and C. Koch. Computational modeling of visual attention. Nat. Rev. Neurosci., 2(3):194-203, 2001.

[2] J. M. Wolfe and T. S. Horowitz. What attributes guide the deployment of visual attention and how do they do it? Nat. Rev. Neurosci., 2004.

[3] A. Yarbus. Eye movements during perception of complex objects. L. Riggs, editor, Eye Movements and Vision, 1967.

[4] M. Land and M. Hayhoe. In what ways do eye movements contribute to everyday activities? Vision Research, 41(25-26):3559-3565, 2001.

[5] M. Hayhoe and D. Ballard. Eye movements in natural behavior. Trends in Cog. Sci., 9(4), 188-193. 2005.

[6] C. Rothkopf, D. Ballard, and, M. Hayhoe. Task and scene context determines where you look. Journal of Vision, 7(14):16, 1-20, 2007.

[7] W., Kienzle, A. F., Wichmann, B., Scholkopf, and M. O. Franz. A nonparametric approach to bottom-up visual saliency. NIPS, 2007.

[8] T. Judd, K. Ehinger, F. Durand and, A. Torralba. Learning to predict where humans look, ICCV, 2009.

[9] E. Vig, M. Dorr, T. Martinetz, and, E. Barth. A learned saliency predictor for dynamic natural scenes, ICANN, LNCS, 6354, 2010.

[10] J. Li, Y. Tian, T. Huang and W. Gao, Probabilistic multi-task learning for visual saliency estimation in video, Int. Journal of Computer Vision, (90)2:150-165, 2010.

[11] A. Kimura, D. Pang, T. Takeuchi, K. Miyazato, J. Yamato and K. Kashino, A stochastic model of human visual attention with a dynamic Bayesian network, IEEE Transactions PAMI. In Press.

[12] M. Hayhoe. Advances in relating eye movements and cognition. Infancy, 6(2): 267-274, 2004.

[13] D. Ballard, M. Hayhoe, and J. Pelz. Memory representations in natural tasks. Journal of Cog. Neurosci., 7(1), 66-80, 1995.

[14] M. F. Land and D. N. Lee. Where we look when we steer. Nature, 369: 742-744, 1994.

[15] R. J. Peters and L. Itti. Beyond bottom-up: Incorporating taskdependent influences into a computational model of spatial attention. CVPR, 2007.

[16] L. Itti, C. Koch, and E. Niebur. A model of saliency-based visual attention for rapid scene analysis. IEEE Transactions PAMI, 20(11):12541259, 1998.

[17] R.J. Peters and L. Itti, Congruence between model and human attention reveals unique signatures of critical visual events. NIPS, 2008

[18] V. Navalpakkam and L. Itti. Modeling the influence of task on attention. Vision Research, 45(2): 205-231, 2005.

[19] N. Sprague, D. H. Ballard, Eye Movements for Reward Maximization NIPS, 2003

[20] H. Hecht, S. Vogt and W. Prinz, Motor learning enhances perceptual judgment: A case for action-perception transfer. Psychological Research, 65:3-14, 2001.

[21] S. Schtz-Bosbach and W. Prinz, Perceptual resonance: Action-induced modulation of perception. Trends in Cog. Sci., 11:349-355, 2007.

[22] G. Rizzolatti, L. Riggio, I. Dascola and, C. Umilt, Reorienting attention across the horizontal and vertical meridians: Evidence in favor of a premotor theory of attention. Neuropsychologia, 1987.

[23] L. Craighero, L. Fadiga , G. Rizzolatti, and C. Umilt, Action for perception: a motor-visual attentional effect. $J$ Exp Psychol Hum Percept Perform, 25(6):1673-92, 1999.

[24] C. Siagian and L. Itti, Rapid biologically-inspired scene classification using features shared with visual attention. IEEE Transactions PAMI, 29(2):300-312, 2007.

[25] N. Pugeault and R. Bowden. Learning pre-attentive driving behavior from holistic visual features. ECCV, 2010.

[26] K. Ehinger, B. Hidalgo-Sotelo, A. Torralba, and A. Oliva. Modelling search for people in 900 scenes: a combined source model of eye guidance. Visual Cognition, 17:945-978, 2009.

[27] S. Chikkerur, T. Serre, C. Tan, and T. Poggio. What and where: a Bayesian inference theory of visual attention. Vision Research, 2010. 